\title{
Optimizing effectiveness and robustness for solar heat absorbers composed of a periodically nano-structured surface
}

\author{
Min-Jhong Gu ${ }^{1} \cdot$ Yu-Bin Chen ${ }^{1,2}$ (D)
}

(c) Springer Nature Switzerland AG 2019

\begin{abstract}
This work studies solar heat absorbers having periodic and rectangular nano-pillars on their surfaces. Design factors are periods, filling ratios, and pillar height. They are tailored using the design of experiments for absorption effectiveness and robustness. The former requires enlarging absorption within the solar spectrum as well as diminishing re-emission loss at long wavelengths. The latter refers to independence of absorption effectiveness from noise interference. Studied noise factors include dimensional deviations and variation of sunlight orientation. Effects from individual design factor and interactions between two design factors are all investigated. The selected absorbers exhibit appealing wavelengthselective absorptance spectra. Absorption effectiveness and robustness of these absorbers are quantitatively illustrated by a large response variable $Q$ and its small variation $S$, respectively.
\end{abstract}

Keywords Absorbers · Design of experiments · Robustness · Solar heat · Wavelength-selective

\begin{tabular}{ll}
\multicolumn{2}{l}{ List of symbols } \\
$d$ & Thickness, $\mathrm{m}$ \\
$E$ & Emissive power, $\mathrm{W} / \mathrm{m}^{2}$ \\
$\overrightarrow{\boldsymbol{E}}$ & Electric field, $\mathrm{V} / \mathrm{m}$ \\
$f$ & Filling ratio \\
$G$ & Irradiation, $\mathrm{W} / \mathrm{m}^{2}$ \\
$I$ & Intensity of radiance, $\mathrm{W} / \mathrm{m}^{2}$ \\
$\vec{k}$ & Wavevector, $\mathrm{m}^{-1}$ \\
$\vec{n}$ & Normal vector \\
$Q$ & Response variable \\
$\bar{Q}$ & Mean of response variable \\
$\mathrm{S}$ & Standard deviation of response variable \\
$W p$ & Watt-peak, $\mathrm{W}$ \\
$w$ & Width, $\mathrm{m}$ \\
$x, y, z$ & Cartesian coordinate system
\end{tabular}

\section{Subscripts}

$1.5 \quad$ AM mass 1.5

$773773 \mathrm{~K}$

i Incident wave

$\begin{array}{ll}t & \text { Total } \\ \text { ideal } & \text { Optimal spectrum } \\ \text { Greek symbols } \\ a & \text { Absorptance } \\ \varepsilon & \text { Emittance } \\ \Lambda & \text { Period, } \mathrm{m} \\ \lambda & \text { Wavelength, } \mathrm{m} \\ \theta & \text { Zenith angle, degree } \\ \phi & \text { Azimuth angle, degree } \\ \psi & \text { Polarization angle, degree }\end{array}$

\section{Introduction}

Solar heat is one promising source of renewable energy thanks to its multiple advantages, such as abundancy, cleanliness, high power density, access easiness, and zero greenhouse gas generation [16]. The heat is generated from our sun and emits out in the form of thermal radiation, i.e., randomly polarized light. The intensity spectrum

$\triangle$ Yu-Bin Chen, ybchen@pme.nthu.edu.tw | 'Department of Power Mechanical Engineering, National Tsing Hua University, No. 101, Section 2, Kuang-Fu Road, Hsinchu 30013, Taiwan. ${ }^{2}$ Department of Mechanical Engineering, National Cheng Kung University, No.1, University Road, Tainan City 70101, Taiwan. 
is close to that from a blackbody surface at $5762 \mathrm{~K}$ following the Planck's law [2]. Its spectral intensity is unevenly distributed, and the bandwidth is wide. Majority of the solar power is within the visible and infrared regions. Total solar power reaching the earth is about $1.7 \times 10^{11} \mathrm{MW}$ [15]. Although the amount is huge, usage of solar heat is not straightforward. The installed global capacity is less than $5 \mathrm{GWp}$ based on recent statistics [18]. One critical issue is the ineffective heat absorption because of concurrent loss from the surface of absorbers. Temperature there is much higher than that of ambient, and the re-emission loss via thermal radiation could be significant. Only wavelengthselective absorbers are able to remedy this drawback [32].

The most popular way of realizing wavelength-selectivity is carefully-tailored surface topography, for example, adding structures over a substrate. Rephaeli and Fan numerically developed a structured tungsten slab with subwavelength periodicity, and the structure consists of a square lattice array of pyramids [26]. Diem et al. [8] added submicron tungsten wires on a silicon nitride substrate to realize a wide-angle perfect absorber. Wu et al. [35] presented a solar heat collection system using a nanoimprint-patterned film of plasmonic structures, which were composed of tungsten rectangular pillars on an aluminum nitride substrate. Wang et al. [34] developed black absorbers using tungsten rectangular nano-structures on a silicon dioxide film and tungsten substrate. In the same year, Hu et al. [14] developed a metamaterial absorber consisting of a chromium circular-shaped ring resonator embedded in a dielectric layer and a chromium ground plane. Cheng et al. [5] presented a photoexcited switchable absorber composed of X-shaped gold structures and a silicon pad. Nunes et al. [24] demonstrated a selective surface composed of black chromium $\left(\mathrm{Cr} / \mathrm{Cr}_{2} \mathrm{O}_{3}\right)$ deposited on substrates of AISI 304 stainless steel for solar heat absorption. Tian et al. proposed a one-dimensional $\mathrm{HfO}_{2} /$ $\mathrm{Al}_{2} \mathrm{O}_{3}-\mathrm{W}$ nanocomposites/W/Al $\mathrm{O}_{3} / \mathrm{W}$ multilayered photonic structure as potential wavelength-selective thermal devices. They theoretically investigated the emission properties of the proposed Mie-resonance metamaterials [31]. Liang et al. [19] recently designed and numerically investigated a dielectric filled two-dimensional nickel grating for wide-angle and polarization-independent absorption in the visible range.

Development of wavelength-selective absorbers has been facilitated with multiple design methodologies. For example, utilizing unique physical mechanisms could enhance absorption at target wavelengths. Successful attempts included generation of interference within a thin film $[2,27,29]$, resonance of an electrical circuit $[7$, $11,20]$, and band structure of photonic crystals $[3,10]$. Additional efforts are necessary for solar heat absorbers using physical mechanisms. These mechanisms need to occur within the broad spectral range of interest.

Other works performed numerical experiments with optimization strategies. One-factor-a-time was intuitive and simple, while the optimization process could end up with a local maximum/minimum easily [27]. Game theory approach was fun and able to identify a satisfactory absorber within a well-defined searching region [13]. Both quality of merit [23] and Taguchi method [4, 17] were utilized to develop absorbers when design objectives were quantitatively defined. On the other hand, the genetic algorithm was capable of tuning all design factors provided computational resources were sufficient [6].

Aforementioned experiments are a series of runs in which purposeful changes are made to the input variables so that reasons for changes are observed in the performance. Each absorber can be visualized as a combination of materials, volume ratio, surface morphology, and micro-/nano-structures that convert sunlight into heat. These variables and material properties are called factors, and some of them are controllable [22]. Two types of factors are often considered in developing absorbers. The design factors are selected for study in the numerical experiments. The noise factors vary naturally and uncontrollably in the process which can be controlled for purposes of an experiment. The conversion efficiency is a common example of design objective.

Previous works mainly studied influences from each of multiple design factors. However, those from an interaction between two design factors were never looked into. Impacts from dimension tolerance and sunlight orientation variation as noise factors were never simultaneously inspected. Performance of developed absorbers then becomes vulnerable to continuously varying sunlight as well as mismatch between designed and real dimensions. The aim of this study is to eliminate above concerns and approach a high-quality solar heat absorber further. Topography of the absorber is defined with five design factors. They are tailored for effective heat absorption, i.e., maximizing the ratio of gained heat over total solar heat. The robustness of performance under noise interferences is taken into account. Studied noise includes the polarization state of incidence, relative orientation between the absorber and direct sunlight, and dimension variation of the absorber. Numerical models are built to evaluate performance of every considered absorber. Subsequently, the design of experiments is employed to quantitatively investigate main effects induced by each design factor and interactions between any two of them. Finally, superiority of developed absorbers on the basis of the absorption effectiveness and robustness is exhibited. 


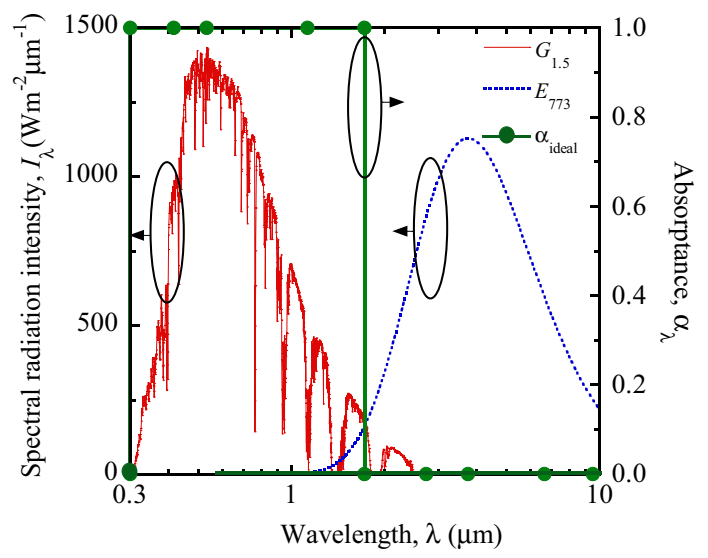

Fig. 1 Direct solar irradiance at $\operatorname{AM} 1.5\left(G_{1.5}\right)$, blackbody radiation spectrum at $T=773 \mathrm{~K}\left(E_{773}\right)$, and wavelength-selective spectral absorptance $\left(a_{\lambda}\right)$ for an ideal solar heat absorber

\section{Design objectives and factors}

Figure 1 plots $G_{1.5}$, the solar irradiation spectrum AM1.5 [30] to specify providing the incident solar heat employed here. The corresponding zenith angle $\theta$ of direct sunlight is $48.2^{\circ}$. Oscillations within the spectrum are caused by absorption and scattering in the atmosphere. Wavelength $\lambda$ of most (>98\%) incident radiation is between 0.3 and $2.1 \mu \mathrm{m}$. Absorbing solar heat within the spectral region becomes the first objective for absorber development. On the other hand, only large absorption is not sufficient to guarantee ideal solar heat absorbers because simultaneous loss from its surface may be also large. The loss is mainly re-emission because temperature of the absorber is much higher than that of ambient [15]. For example, the surface temperature is $773 \mathrm{~K}$, melting temperature of a phase change material $\mathrm{CaCl}_{2} / \mathrm{NaCl}$ [1]. The material directly contacts the absorber and saves absorbed energy using the latent heat. Reducing the re-emission loss is also needed for absorption effectiveness. For demonstration convenience, $773 \mathrm{~K}$ is assigned as the absorber temperature hereafter. Figure 1 also plots spectral emissive power from a blackbody surface at $773 \mathrm{~K}\left(E_{773}\right)$. The two spectra, $G_{1.5}$ and $E_{773}$, cross each other at the wavelength $\lambda=1.73 \mu \mathrm{m}$. Spectral power of incoming solar heat is larger than emission loss at short wavelengths, but emission loss is relatively large at long wavelengths. Therefore, spectral absorptance of an ideal absorber is unity $\left(a_{\lambda}=1\right)$ at $\lambda<1.73$ $\mu \mathrm{m}$ for large absorption. The spectral emittance is null $\left(\varepsilon_{\lambda}=0\right)$ at $\lambda>1.73 \mu \mathrm{m}$ for little re-emission. The spectral absorptance of an ideal solar heat absorber is plotted as $a_{\text {ideal. }}$. Notice that Kirchhoff's law [2] is applied here.

Figure 2 shows proposed topography for solar heat absorbers. The absorber surface contains two-dimensionally periodic rectangular structures. Defining their

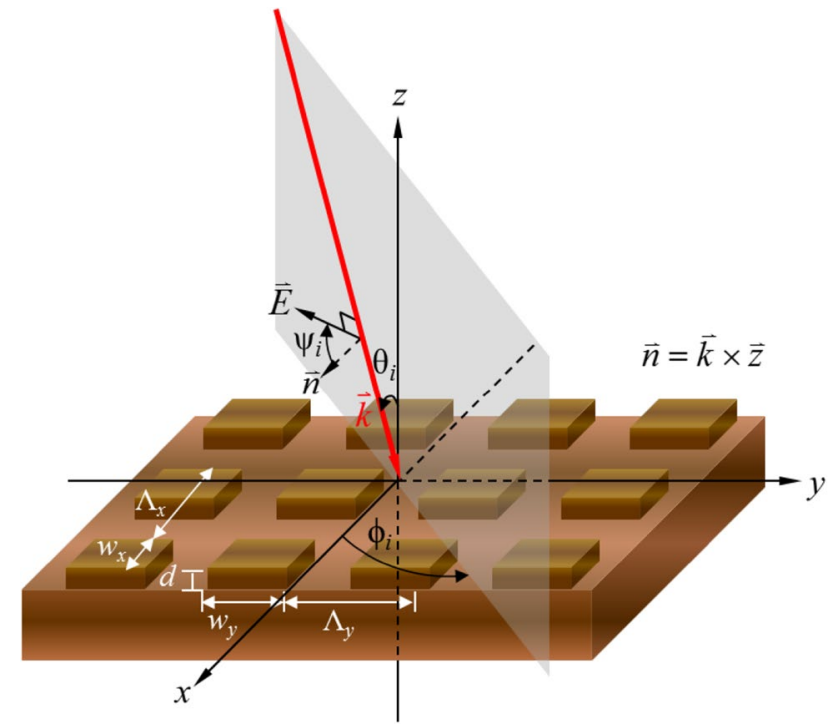

Fig. 2 Schematic of proposed absorber under incidence. The absorber surface contains periodic rectangular structures. The incidence direction of place wave is defined with the zenith angle $\left(\theta_{i}\right)$ and azimuth angle $\left(\phi_{i}\right)$, while polarization state is specified with the polarization angle $\left(\psi_{i}\right)$

dimensions along the $z$-axis is thickness $d$. On the other hand, $\Lambda_{x}$ and $\Lambda_{y}$ are periods along the $x$ - and $y$-axis, respectively. Corresponding filling ratios along the $x$ - and $y$-axis are $f_{x}$ and $f_{y}$, respectively. The filling ratio $f$ is defined by ratio between the side length of a nano-structure $w$ and period $\Lambda$, i.e., $f=w / \Lambda$. These five dimensions are design factors that are tailored for absorption effectiveness and robustness. A dimensional error is a noise factor possibly caused by fabrication tolerance or thermal expansion. Schematic of directly incident sunlight is also shown in Fig. 2. Orientation of the sunlight is defined using the zenith angle $\theta_{i}$ and azimuth angle $\phi_{i}$, where subscript $i$ refers incident wave. Polarization state is determined by the polarization angle $\psi_{i}$. It is the angle between electric field $\overrightarrow{\boldsymbol{E}}$ and normal vector $\vec{n}$ on the plane of incidence. Because all of three angles are varying constantly and naturally, they are included as noise factors, too.

Copper is selected as the absorber material because of its high temperature sustainability, thermal conductivity [2], and fabrication easiness. In fact, Cu nano-structures have recently exhibited promising values for solar heat absorbers [9, 33]. Wavelength-dependent optical constants of $\mathrm{Cu}$ in Ref. [25] after appropriate interpolation were employed in our numerical model. Only the spectral range from 0.3 to $9.52 \mu \mathrm{m}$ is taken into account for emission loss because of limited data availability. Emitting power within the spectral range is $82.7 \%$ for $E_{773}$. The portion is sufficient for performance evaluation. In the following numerical experiments, spectral absorptance $a_{\lambda}$ is 
obtained from programs based on the rigorous coupledwave analysis [4, 12]. Inputs include both incidence and absorber topography, while convergence of programs have been re-validated.

Absorption effectiveness and robustness are our two design objectives. The absorption effectiveness of a solar heat absorber was often evaluated using total absorptance $a_{t}[21]$ as given in the following:

$\alpha_{t}=\frac{\int_{0}^{\infty} \alpha_{\lambda} G_{1.5} d \lambda}{\int_{0}^{\infty} G_{1.5} d \lambda}$

where the numerator and denominator are the whole absorbed heat and all incident solar heat, respectively. But $a_{t}$ in Eq. (1) does not count the loss from re-emission and is impractical due to unavailability of $a_{\lambda}$ at extremely short or long wavelengths. As a result, quantitative evaluation on absorption effectiveness of absorbers at $773 \mathrm{~K}$ in this work utilizes the response variable $Q$ below:

$Q=\frac{\int_{0.3 \mu \mathrm{m}}^{9.52 \mu \mathrm{m}} \alpha_{\lambda}\left(G_{1.5}-E_{773}\right) d \lambda}{\int_{0.3 \mu \mathrm{m}}^{9.52 \mu \mathrm{m}} G_{1.5} d \lambda}$

where the numerator and denominator are the net absorbed heat and incident solar heat, respectively. Integrations are replaced with summation in our work without losing accuracy. $Q$ is a dimensionless number determined by $a_{\lambda}$ of each absorber. The minimum and maximum of $a_{\lambda}$ is -5.47 and 0.98 , respectively. A positive and large $Q$ illustrates that the absorber gains solar heat effectively. And it can be modified for absorbers working at temperature other than $773 \mathrm{~K}$.

Table 1 lists controllable design factors and their levels employed in our design of experiments. Five control factors $\mathbf{A}, \mathbf{B}, \mathbf{C}, \mathbf{D}$, and $\mathbf{E}$ are $\Lambda_{x^{\prime}} \Lambda_{y^{\prime}} f_{x^{\prime}} f_{y^{\prime}}$ and $d$, respectively. Each factor has three levels for optimization, while defining these levels considers fabrication feasibility and sufficient coverage. Levels of factor $\mathbf{A}$ and $\mathbf{B}$ both are $300 \mathrm{~nm}$, $1200 \mathrm{~nm}$, and $2100 \mathrm{~nm}$. The minimum $(\Lambda=300 \mathrm{~nm})$ is identical to the shortest wavelength of interest. The maximum

Table 1 Controllable design factors and their levels in design of experiments

\begin{tabular}{lllll}
\hline No. & Design factor & \multicolumn{2}{l}{ Level } \\
\cline { 3 - 5 } & & $0 /-$ & $1 /$ N. A. & $2 /+$ \\
\hline A & $\Lambda_{x}(\mathrm{~nm})$ & 300 & 1200 & 2100 \\
B & $\Lambda_{y}(\mathrm{~nm})$ & 300 & 1200 & 2100 \\
C & $f_{x}$ & 0.3 & 0.6 & 0.9 \\
D & $f_{y}$ & 0.3 & 0.6 & 0.9 \\
E & $d(\mathrm{~nm})$ & 200 & 500 & 800 \\
\hline
\end{tabular}

$(\Lambda=2100 \mathrm{~nm})$ is the same as upper wavelength limit of $G_{1.5}$. They are correlated to wavelengths because tailoring absorptance is often realized using structure size comparable to radiation wavelengths. Middle value of these two periods $(\Lambda=1200 \mathrm{~nm})$ is the other level of both factors. Levels for $\mathbf{C}$ and $\mathbf{D}$ share $0.3,0.6$, and 0.9. On the other hand, levels for $\mathbf{E}$ are $200 \mathrm{~nm}, 500 \mathrm{~nm}$, and $800 \mathrm{~nm}$. Notice that the feasibility of nano-structures is taken into account when levels are selected.

Table 2 shows five noise factors and their three levels. They are studied for absorption robustness of optimized absorbers. These noises originate from dimensional errors and essence of sunlight. The former brings relative period error $\mathbf{a}(\Delta \Lambda / \Lambda)$, filling ratio error $\mathbf{b}(\Delta f)$, and relative thickness error $\mathbf{c}(\Delta d / d)$. Three levels for each noise are $-0.1,0$, and 0.1. All of noise $\mathbf{d}, \mathbf{e}$, and $\mathbf{f}$ are angles, but their levels are different. The levels of noise $\mathbf{d}$ (zenith angle, $\theta_{j}$ ) are $0^{\circ}$, $30^{\circ}$, and $60^{\circ}$ because $\theta_{i}$ decreases from $90^{\circ}$ (sunrise) monotonically to $0^{\circ}$ and then increases to $90^{\circ}$ (sunset). Each level covers about equal day time. On the other hand, the three levels of noise e (azimuthal angle, $\phi_{i}$ ) are $0^{\circ}, 45^{\circ}$, and $90^{\circ}$. Although $\phi_{i}$ ranges from $0^{\circ}$ to $360^{\circ}$, symmetry of four quadrants has been considered in defining levels. $\phi_{i}=0^{\circ}$ and $\phi_{i}=90^{\circ}$ are associated with the solar motion on the $y-z$ and $x-z$ planes, respectively. $\phi_{i}=45^{\circ}$ refers to the third plane $(x=y)$, which equally splits the aforementioned two planes. The noise $\mathbf{f}$ (polarization angle, $\psi_{i}$ ) symbolizes the polarization of incidence. The TM (transverse magnetic) and TE (transverse electric) waves are at $\psi_{i}=0^{\circ}$ and $\psi_{i}=90^{\circ}$, respectively. These two are extremes of polarization state for an electromagnetic wave, and $\psi_{i}=45^{\circ}$ refers to middle state between the two.

\section{Screening experiments}

Conducting screening experiments is to identify factor(s) significantly influencing the absorption effectiveness and robustness. The screening experiments can also study impacts from two-factor interactions [28]. Base for optimization experiments is then set up, and Table 3 lists an

Table 2 Noise factors and their levels in design of experiments

\begin{tabular}{lllll}
\hline No. & Noise factor & \multicolumn{2}{l}{ Level } \\
\cline { 3 - 5 } & & 0 & 1 & 2 \\
\hline $\mathrm{a}$ & $\Delta N \Lambda$ & -0.1 & 0 & 0.1 \\
$\mathrm{~b}$ & $\Delta f$ & -0.1 & 0 & 0.1 \\
$\mathrm{c}$ & $\Delta d / d$ & -0.1 & 0 & 0.1 \\
$\mathrm{~d}$ & $\theta_{i}$ (degree) & 0 & 30 & 60 \\
$\mathrm{e}$ & $\phi_{i}$ (degree) & 0 & 45 & 90 \\
$\mathrm{f}$ & $\psi_{i}$ (degree) & 0 & 45 & 90
\end{tabular}


orthogonal table for our screening experiments. Total 16 experiments are performed according to a $2^{5-1}$ fractional factorial design with an accuracy of $\mathbf{V}(5)$. The correlation $\mathbf{E}=\mathbf{A B C D}$ is assigned to five design factors here for reducing calculation loading. Thereafter, interferences from noise factors are added to obtain the response variable $Q$. A fractional factorial design of $3^{6-4}$ with an accuracy of III(3) is constructed using four relationships among noises. The relationships are $\mathbf{c}=\bmod (2 \mathbf{a}+\mathbf{b}, 3), \mathbf{d}=\bmod (\mathbf{b}+\mathbf{c}, 3)$, $\mathbf{e}=\bmod (\mathbf{c}+\mathbf{d}, 3)$, and $\mathbf{f}=\bmod (\mathbf{d}+2 \mathbf{e}, 3)$ Rushing et al. [28]. As a result, 9 sets of environment are ready for programs to obtain $Q$ from each of the aforementioned 16 experiments. The average $\bar{Q}$ over 9 Q's is able to diminish bias on the response variable in reality. Furthermore, variation among these $Q$ 's provides an estimate of performance variation. $\bar{Q}$ and the standard deviation $S$ are also listed in Table 3 for further analysis. For example, the mean effect of factor $\mathbf{A}$ is the difference between the average of $Q$ from even number of experiments and the counterpart from odd number of experiments. Its value is $-0.05=[(0.18-0$. $38-0.39+0.13-1.14+0.10+0.10-0.55)-(0.22+0.17+0$. $21-1.21+0.14-0.17-0.94+0.08)] / 8$. The standard deviation for factor $\mathbf{A}$ is calculated using -0.05 and $\bar{Q}$. Similarly, effect of another factor or any two-factor interaction can also be calculated.

Table 4 lists the mean effects and standard deviation of each. Left columns show those from a design factor, while right columns specify those from two-factor interactions. Overall, $|\bar{Q}|$ (magnitudes of $\bar{Q}$ ) from the design factor $\mathbf{E}$, the two-factor interaction $\mathbf{A C}$, and the two-factor interaction BD are larger than others. They dominantly influence $Q$ of proposed absorbers. The negative $\bar{Q}$ from $\mathbf{E}$ means that $Q$ gets small as the level of $\mathbf{E}$ increases. The positive $\bar{Q}$ from
Table 4 Effect estimated from the screening experiment

\begin{tabular}{llrlrrr}
\hline Design factor & Effect & & $\begin{array}{l}\text { Two-factor } \\
\text { interaction }\end{array}$ & \multicolumn{2}{l}{ Effect } \\
\cline { 2 - 3 } & & & & & \\
\cline { 2 - 3 } & $\bar{Q}$ & $S$ & & $\bar{Q}$ & \multicolumn{1}{c}{$S$} \\
\hline A & -0.05 & -0.02 & AB & 0.16 & -0.14 \\
B & -0.03 & -0.08 & AC & 0.34 & -0.27 \\
C & -0.21 & 0.13 & AD & -0.09 & 0.13 \\
D & -0.16 & 0.21 & AE & -0.03 & 0.03 \\
E & -0.71 & 0.50 & BC & -0.11 & 0.17 \\
& & & BD & 0.35 & -0.26 \\
& & & BE & 0.01 & -0.03 \\
& & & CD & 0.15 & -0.15 \\
& & & CE & -0.19 & 0.16 \\
& & & DE & -0.10 & 0.17 \\
\hline
\end{tabular}

AC means that $Q$ enlarges when levels of factors $\mathbf{A}$ and $\mathbf{C}$ simultaneously increase or decrease. Conversely, $Q$ diminishes when a level of the two factors increases while the other decreases. The largest magnitude of $S$ is caused by factor $\mathbf{E}$ only. As the level of factor $\mathbf{E}$ increases, variation of $Q$ gets significant.

Figure 3 shows semi-normal probability plot of effects from screening experiments. Magnitudes of both effects, $|\bar{Q}|$ and $|S|$, from each factor and two-factor interactions are marked. Dashed lines are regression curves with a cumulative probability less than $75 \%$. The corresponding factor or interaction is not dominant to an effect if the mark is nearby the curve. For an outlier away from the regression curve, its corresponding factor or interaction influences the effect significantly. Accordingly, the factor $\mathbf{E}$, interaction $\mathbf{A C}$, and interaction $\mathbf{B D}$ all strongly influence $|\bar{Q}|$. One
Table 3 Construction of the $2_{\mathrm{v}}^{5-1}$ design with the defining relation $\mathbf{E}=\mathbf{A B C D}$

\begin{tabular}{llllllrl}
\hline Exp. & A & B & C & D & E & Q & \multicolumn{1}{c}{$S$} \\
\hline 1 & - & - & - & - & + & 0.22 & 0.06 \\
2 & + & - & - & - & - & 0.18 & 0.12 \\
3 & - & + & - & - & - & 0.17 & 0.07 \\
4 & + & + & - & - & + & -0.38 & 0.20 \\
5 & - & - & + & - & - & 0.21 & 0.11 \\
6 & + & - & + & - & + & -0.39 & 0.35 \\
7 & - & + & + & - & + & -1.21 & 1.06 \\
8 & + & + & + & - & - & 0.13 & 0.04 \\
9 & - & - & - & + & - & 0.14 & 0.22 \\
10 & + & - & - & + & + & -1.14 & 1.27 \\
11 & - & + & - & + & + & -0.17 & 0.31 \\
12 & + & + & - & + & - & 0.10 & 0.09 \\
13 & - & - & + & + & + & -0.94 & 0.96 \\
14 & + & - & + & + & - & 0.10 & 0.09 \\
15 & - & + & + & + & - & 0.08 & 0.13 \\
16 & + & + & + & + & + & -0.55 & 0.63 \\
\hline
\end{tabular}




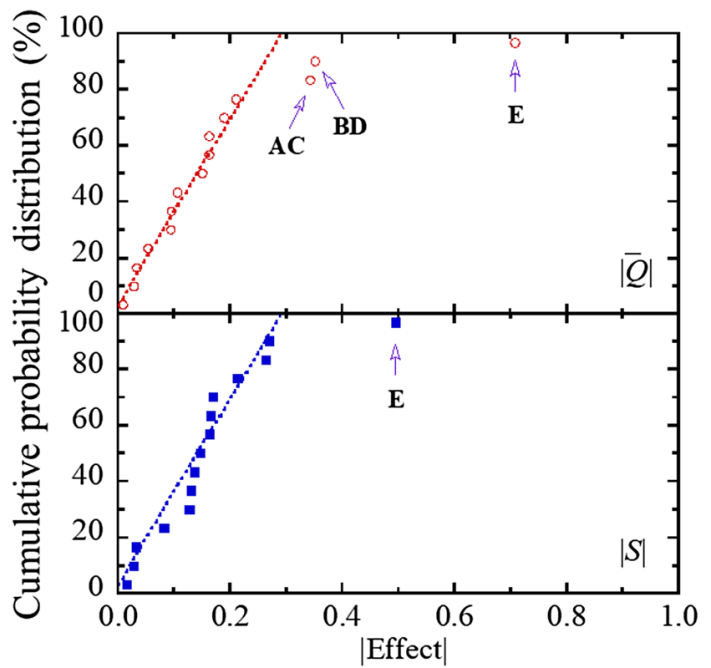

Fig. 3 Normal probability plot of effects from the screening experiment

may confuse that none of $\mathbf{A}, \mathbf{B}, \mathbf{C}$, or $\mathbf{D}$ can lay impact on $|\bar{Q}|$, while their interaction can. The confusion can be easily clarified once the absorber surface profile is recalled. The two-factor interaction is actually $w$, the side length of nano-structure. $w_{x}$ is determined only when levels of $\mathbf{A}$ and $\mathbf{C}$ are both set. The same explanation can be applied to interaction BD. For $|S|, \mathbf{E}$ is the only critical factor. When the level of $\mathbf{E}$ increases, variation of absorption effectiveness enlarges. Influences from other factors or interactions are relatively trivial. To sum up, absorption effectiveness and robustness of the proposed absorbers are dominated by $\mathbf{E}, \mathbf{A C}$, and $\mathbf{B D}$. The next step is to perform optimization experiments using two sets of factors, ACE and BDE.

\section{Optimization experiments}

Figure $4 a$ and $b$ show two main effects $\bar{Q}$ and $S$ from optimization experiments using $\mathrm{ACE}$ and $\mathrm{BDE}$, respectively. Each experiment employs a full factorial design [22], and each factor has three levels. A data point is the average of effects from experiments with the same level for a specified factor. Three points are obtained, and they are then connected to display a non-linear relationships between an effect and design factor. A dashed horizontal line serves as a benchmark. It is the average effect from all of the experiments. Large deviation between a solid line and the dashed line symbolizes dominance of a design factor on the effect.

Figure 4a shows that $\bar{Q}$ decreases monotonically and significantly with levels of $\mathbf{E}$. But the correlation between $\bar{Q}$ and $\mathbf{A}$ is trivial as the correlation between $\bar{Q}$ and $\mathbf{C}$. The difference between $\mathbf{E}$ and the other two design factors
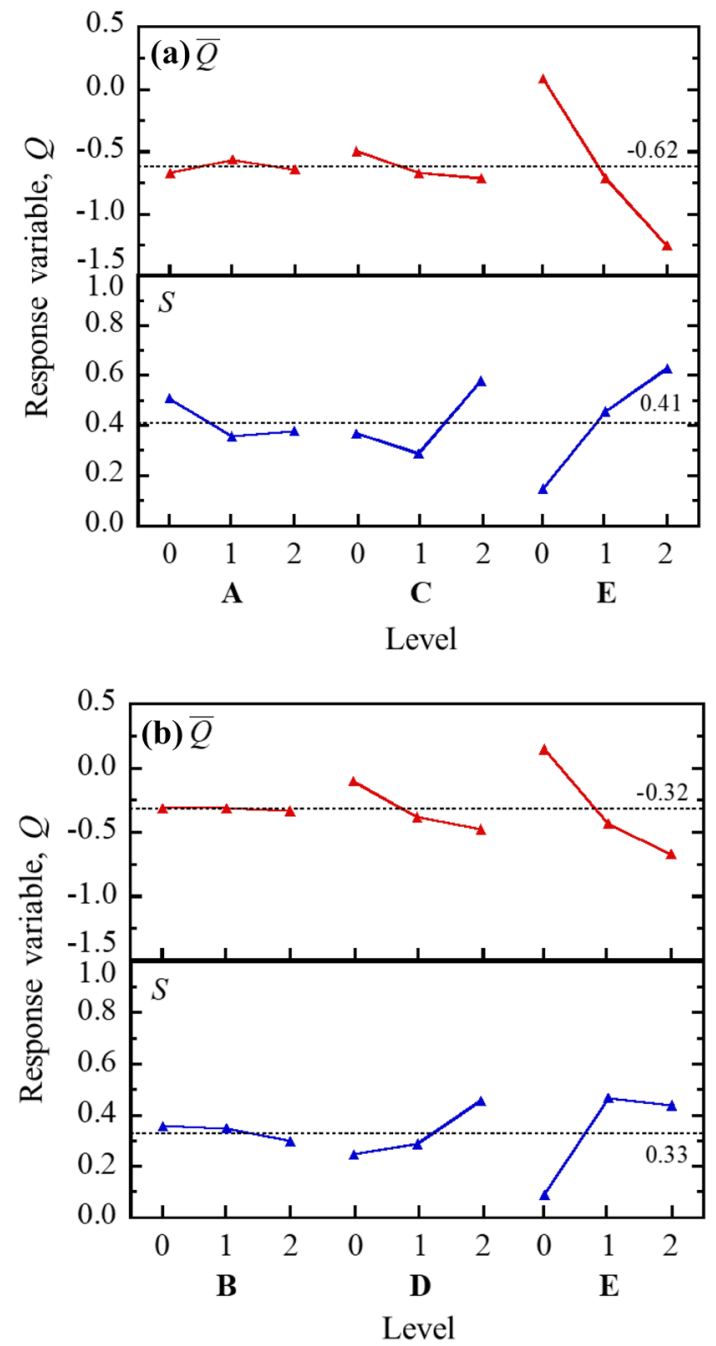

Fig. 4 Main effects from optimization experiments: a ACE, b BDE

assures the single dominance of $\mathbf{E}$ on $\bar{Q}$. Similarly, $\mathbf{E}$ is the only dominant factor on $S$. But $S$ monotonically increases with levels of $\mathbf{E}$. Figure $4 \mathrm{~b}$ also shows most of the aforementioned findings. The only exception is the correlation between $\mathbf{E}$ and $S$. As level of $\mathbf{E}$ increases from 1 to $2, S$ decreases a little. Because absorption effectiveness desires a large $\bar{Q}$ and absorption robustness needs a low $S$, the optimized level of $\mathbf{E}$ is 0 .

Figure 5 shows influences on main effects from two-factor interactions. Influences from $\mathbf{A C}, \mathbf{A E}$, and $\mathbf{C E}$ are plotted in Fig. 5a. Those from BD, BE, and DE are shown in Fig. 5b. A data point is obtained when levels of two involved factors are assigned. Three points are marked identically when the same level is assigned to a factor. Points are then connected with straight line sections to display correlations between an interaction and an effect. For instance, lines connecting red triangles exhibit influences from AC as the level of $\mathbf{C}$ is fixed to 0 . At the same time, the level of 


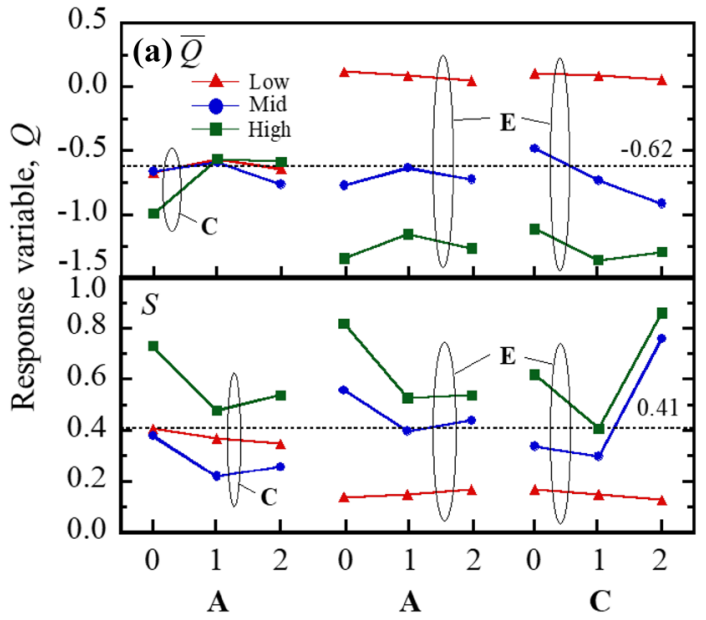

Level

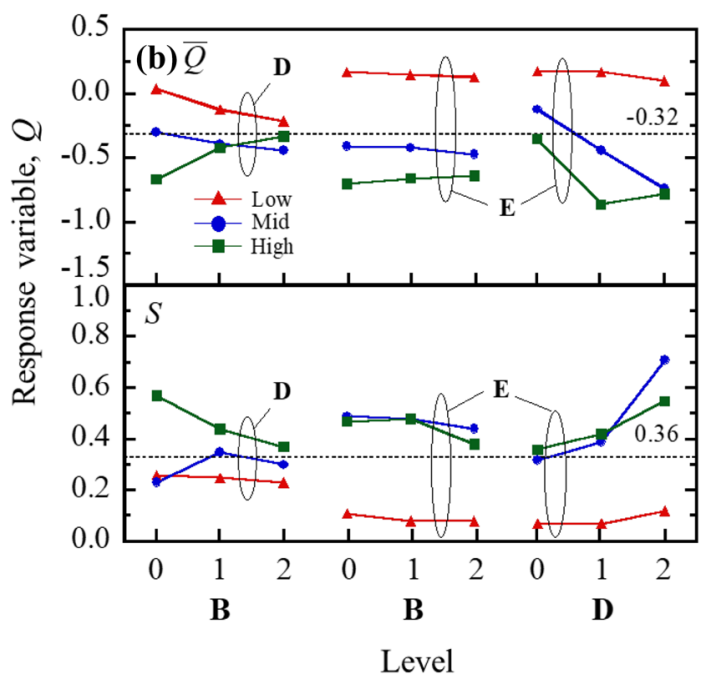

Fig. 5 Interaction effects from optimization experiments: a ACE, b BDE

factor $\mathbf{A}$ varies from 0 to 2 . If lines cross each other, $\mathbf{A C}$ is strong enough to influence the main effect. Otherwise, the two-factor interaction is trivial. Examples include interactions $\mathbf{A E}$ and $\mathbf{C E}$ when the level of $\mathbf{E}$ is $\mathbf{0}$. Furthermore, connected lines in this example are close to horizontal lines. Changes in main effects are negligible regardless of $\mathbf{A}$ and C. Such little dependence on level of an insensitive factor will be investigated later.

Plots of AC and counterparts of BD both show crossings. These crossings confirm dominant influences from AC and BD on $Q$. These plots further help selecting the best level for the four factors. Firstly, the largest $\bar{Q}$ and smallest $S$ are generated when levels of $\mathbf{A}$ and $\mathbf{C}$ are both 1 (middle level). Secondly, the largest $\bar{Q}$ occurs if levels of $\mathbf{B}$ and $\mathbf{D}$ are both 0 (low level). As a result, optimized levels for $\mathbf{A}, \mathbf{B}, \mathbf{C}, \mathbf{D}$, and $\mathbf{E}$ of the ideal absorber are 1, $0,1,0$, and 0 , respectively. In our following discussion, these optimized levels define the absorber considered in Case 1. Its detailed $a_{\lambda}$ spectra under noise interferences will be plotted and compared with those of a flat plate as well as absorbers in another two cases. Case 2 examines if an insensitive factor $\mathbf{A}$ can provide dimensional tolerance without much deteriorating absorber performance. The proposed absorber sets level 0 for $\mathbf{A}$, while the other four factors are identical to those of the absorber in Case 1. Case 3 looks into influences from the dominant design factor $\mathbf{E}$. The proposed absorber sets level 1 for $\mathbf{E}$, while the other four factors are the same as those of the absorber in Case 1.

Figure 6 plots $a_{\lambda}$ within the spectral range of $0.3 \mu \mathrm{m} \leq \lambda \leq 9.52 \mu \mathrm{m}$ for a flat copper plate as well as absorbers in Cases 1, 2, and 3. Each sub-figure shows results of numerical experiments under interference from a set of noise factors. Because total nine sets are investigated, the number of sub-figure is nine. Insects in each specify $Q$ of the four absorbers. In general, $Q$ of absorbers in Cases 1 and 2 is larger than that of a flat $\mathrm{Cu}$ plate. The well-designed absorbers indeed can outperform a plate under various noise interference. But if an absorber is not well designed, nano-structured surfaces may be worse than a plate. For example, the absorber in Case 3 is not suitable for solar heat application. Because the profile difference between absorbers in Case 1 and Case 3 is just a level of a factor, caution really needs to be paid during profile optimization.

Table 5 lists the mean and standard deviation of response variable $Q$ in Fig. 6 as $\bar{Q}$ and $S$, respectively. The first finding is that $\bar{Q}$ for Cases 1 and 2 are both large. Absorbers in the two cases can acquire solar heat more effectively than the other two. Second, because $S$ in the two cases are smaller than that in Case 3, absorption robustness is assured. Third, both $\bar{Q}$ and $S$ are larger in Case 2 than in Case 1. The absorber in Case 2 can acquire solar heat more effectively than that in Case 1. But the absorber in Case 1 can resist noise interferences better than that in Case 2. Due to this trade-off, the two absorbers are regarded equally satisfactory on the basis of absorption effectiveness and robustness. Since both absorbers perform well, design flexibility from the insensitive factor $\mathbf{A}$ is also confirmed. Both level 0 and level 1 of $\mathbf{A}$ can give a good solar heat absorber. Fourth, $\bar{Q}$ becomes negative in Case 3. This negative value implies that re-emission loss is larger than absorbed heat for the absorber. Its temperature cannot maintain at $773 \mathrm{~K}$ in this case. Fifth, $S$ for Case 3 is larger than that for Case 1 because shifting the level of $\mathbf{E}$ from 0 to 1 deteriorates absorption robustness. Nano-structures need to be carefully designed. Last but not least, the smallest $S$ among four absorbers comes from the flat plate. One reason is null interference from noises $\mathbf{a}, \mathbf{b}$, and $\mathbf{c}$. The other is 


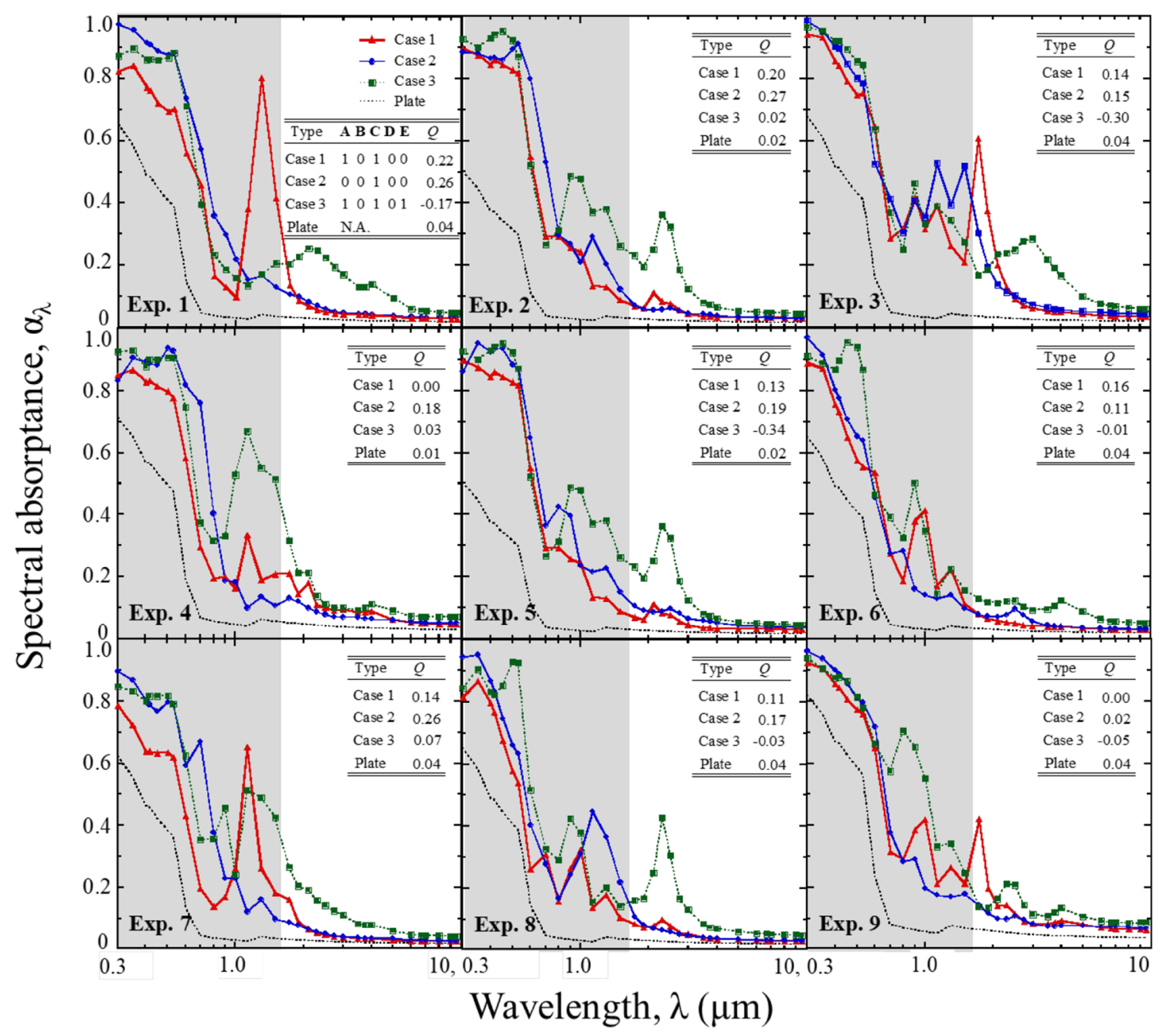

Fig. 6 Spectral absorptance of a flat copper plate, the optimal absorber in Case $1(\mathbf{A B C D E}=10100)$, absorber in Case $2(\mathbf{A B C D E}=00100)$, and absorber in Case $3(\mathbf{A B C D E}=10101)$

Table 5 Effect estimated from nine spectral absorptance spectra in Fig. 6

\begin{tabular}{llllllll}
\hline Type & A & B & C & D & E & Q & S \\
\hline Case 1 & 1 & 0 & 1 & 0 & 0 & 0.122 & 0.077 \\
Case 2 & 0 & 0 & 1 & 0 & 0 & 0.179 & 0.081 \\
Case 3 & 1 & 0 & 1 & 0 & 1 & -0.087 & 0.149 \\
Plate & N.A. & N.A. & N.A. & N.A. & N.A. & 0.028 & 0.016 \\
\hline
\end{tabular}

weak interferences from noise $\mathbf{d}, \mathbf{e}$, and $\mathbf{f}$ thanks to isotropic surface morphology.

\section{Conclusions}

This work successfully developed solar heat absorbers via design of experiments. Settings of five controllable design factors are optimized, and variability transmitted from six noise factors are minimized. Critical impacts come from the structure thickness as well as the interaction between length and width. Studied noise factors include sunrise and sunset $\left(\theta_{i}\right)$, relative orientation between the sun and absorber $\left(\phi_{i}\right)$, incident light polarization state $\left(\psi_{i}\right)$, and dimensional errors $(\Delta \Lambda / \Lambda, \Delta f$, and $\Delta d / d)$. Topography of two satisfactory absorbers is identified after numerical experiments and optimization. One absorber has better absorption effectiveness, while the other has better robustness. These absorbers are composed of periodic and rectangular Cu nano-structures to facilitate 
absorbing, transferring, and even storing thermal energy. Their absorption effectiveness and robustness are quantitatively validated. The same design methodology can facilitate development of absorbers using other materials or working at temperature different from $773 \mathrm{~K}$. These absorbers shall boost utilization of solar heat and diminish consumption of fossil fuels.

Acknowledgements The authors appreciate the Ministry of Science and Technology (MOST) in Taiwan for financial supports under Grant Nos. MOST 107-2218-E-007-016, MOST 106-2628-E-007-006-MY3, and MOST 107-2622-E-007-014-CC2. They also thank Mr. Nitesh Anand and Mr. Swami Siddharth in proofreading English during revision.

Funding The study was funded by the Ministry of Science and Technology (MOST) of Taiwan. The funding Grant No. are: MOST 107-2218-E-007-016, MOST 106-2628-E-007-006-MY3, and MOST 107-2622-E-007-014-CC2.

\section{Compliance with ethical standards}

Conflict of interest On behalf of all authors, the corresponding author states that there is no conflict of interest.

Human and animal rights This research does not involve any Human Participants and/or Animals.

\section{Appendix}

Construction of the $3^{6-4}$ design with the defining relation $\mathbf{c}=\bmod (2 \mathbf{a}+\mathbf{b}, 3), \mathbf{d}=\bmod (\mathbf{b}+\mathbf{c}, 3), \mathbf{e}=\bmod (\mathbf{c}+\mathbf{d}, 3)$ and $\mathbf{f}=\bmod (\mathbf{d}+2 \mathbf{e}, 3)$.

\begin{tabular}{lllllll}
\hline Exp. & A & B & C & D & E & F \\
\hline 1 & 0 & 0 & 0 & 0 & 0 & 0 \\
2 & 0 & 1 & 1 & 2 & 0 & 2 \\
3 & 0 & 2 & 2 & 1 & 0 & 1 \\
4 & 1 & 0 & 2 & 2 & 1 & 1 \\
5 & 1 & 1 & 0 & 1 & 1 & 0 \\
6 & 1 & 2 & 1 & 0 & 1 & 2 \\
7 & 2 & 0 & 1 & 1 & 2 & 2 \\
8 & 2 & 1 & 2 & 0 & 2 & 1 \\
9 & 2 & 2 & 0 & 2 & 2 & 0 \\
\hline
\end{tabular}

\section{References}

1. Alva G, Liu L, Huang X, Fang G (2017) Thermal energy storage materials and systems for solar energy applications. Renew Sust Energ Rev 68(1):693-706. https://doi.org/10.1016/j. rser.2016.10.021

2. Bergman TL, Incropera FP, DeWitt DP, Lavine AS (2011) Fundamentals of heat and mass transfer. Wiley, New York
3. Bermel P, Ghebrebrhan M, Harradon M, Yeng YX, Celanovic I, Joannopoulos JD, Soljacic M (2011) Tailoring photonic metamaterial resonances for thermal radiation. Nanoscale Res Lett 6(1):549. https://doi.org/10.1186/1556-276X-6-549

4. Chen YB, Tan KH (2010) The profile optimization of periodic nano-structures for wavelength-selective thermophotovoltaic emitters. Int J Heat Mass Transfer 53(23-24):5542-5551. https ://doi.org/10.1016/j.ijheatmasstransfer.2010.06.051

5. Cheng Y, Gong R, Zhao J (2016) A photoexcited switchable perfect metamaterial absorber/reflector with polarizationindependent and wide-angle for terahertz waves. Opt Mater 62:28-33. https://doi.org/10.1016/j.optmat.2016.09.042

6. Choi J, Kim M, Kang K, Lee I, Lee BJ (2018) Robust optimization of a tandem grating solar thermal absorber. J Quant Spectrosc Ra 209:129-136. https://doi.org/10.1016/j.jqsrt.2018.01.028

7. Costa F, Genovesi S, Monorchio A, Manara G (2013) A circuitbased model for the interpretation of perfect metamaterial absorbers. IEEE Trans Antennas Propag 61(3):1201-1209. https ://doi.org/10.1109/TAP.2012.2227923

8. Diem M, Koschny T, Soukoulis CM (2009) Wide-angle perfect absorber/thermal emitter in the terahertz regime. Phys Rev B 79(3):033101. https://doi.org/10.1103/PhysRevB.79.033101

9. Fan $\mathrm{P}, \mathrm{Wu} \mathrm{H}$, Zhong $\mathrm{M}$, Zhang H, Bai B, Jin G (2016) Large-scale cauliflower-shaped hierarchical copper nanostructures for efficient photothermal conversion. Nanoscale 8(30):1461714624. https://doi.org/10.1039/C6NR03662G

10. Florescu M, Lee H, Stimpson AJ, Dowling J (2005) Thermal emission and absorption of radiation in finite inverted-opal photonic crystals. Phys Rev A 72:033821-033829. https://doi. org/10.1103/PhysRevA.72.033821

11. Ghosh S, Bhattacharyya S, Kaiprath Y, Srivastava KV (2014) Bandwidth-enhanced polarization-insensitive microwave metamaterial absorber and its equivalent circuit model. J Appl Phys 115(10):104503. https://doi.org/10.1063/1.4868577

12. Ho CC, Chen YB, Shih FY (2016) Tailoring broadband radiative properties of glass with silver nano-pillars for saving energy. Int J Therm Sci 102:17-25. https://doi.org/10.1016/j.ijthermals ci.2015.10.038

13. Hu Y, Rao SS (2009) Game-theory approach for multi-objective optimal design of stationary flat-plate solar collectors. Eng Optim 41(11):1017-1035. https://doi.org/10.1080/03052 150902890064

14. Hu D, Wang HY, Zhu QF (2016) Design of an ultra-broadband and polarization-insensitive solar absorber using a circularshaped ring resonator. J Nanophotonics 10(2):02621. https:// doi.org/10.1117/1.JNP.10.026021

15. Kalogirou SA (2004) Solar thermal collectors and applications. Prog Energy Combust Sci 30(3):231-295. https://doi. org/10.1016/j.pecs.2004.02.001

16. Kannan N, Vakeesan D (2016) Solar energy for future worlda review. Renew Sust Energ Rev 62:1092-1105. https://doi. org/10.1016/j.rser.2016.05.022

17. Lee BJ, Chen YB, Han S, Chiu FC, Lee HJ (2014) Wavelengthselective solar thermal absorber with two-dimensional nickel gratings. J Heat Transfer 136(7):072702. https://doi. org/10.1115/1.4026954

18. Lewis NS (2016) Research opportunities to advance solar energy utilization. Science 351:aad.1920. https://doi. org/10.1126/science.aad1920

19. Liang $Q$, Duan H, Zhu X, Chen X, Xia X (2019) Solar thermal absorber based on dielectric filled two-dimensional nickel grating. Opt Mat Express 9(8):3193-3203. https://doi. org/10.1364/OME.9.003193

20. Luo H, Wang T, Gong RZ, Nie Y, Wang X (2011) Extending the bandwidth of electric ring resonator metamaterial absorber. 
Chin Phys Lett 28(3):034204. https://doi.org/10.1088/0256307X/28/3/034204

21. Modest MF (2013) Radiative heat transfer, 3rd edn. Academic Press, New York

22. Montgomery DC (2013) Design and analysis of experiments, 8th edn. Wiley, Hoboken, NJ

23. Nam Y, Yeng YX, Lenert A, Bermel P, Celanovic I, Soljačić M, Wang EN (2014) Solar thermophotovoltaic energy conversion systems with two-dimensional tantalum photonic crystal absorbers and emitters. Sol Energy Mater Sol Cells 122:287-296. https://doi. org/10.1016/j.solmat.2013.12.012

24. Nunes RAX, Costa VC, Sade W, Araújo FR, Silva GM (2018) Selective surfaces of black chromium for use in solar absorbers. Mater Res 21(1):e20170556. https://doi. org/10.1590/1980-5373-mr-2017-0556

25. Palik ED (1998) Handbook of optical constants of solids. Academic Press, Cambridge

26. Rephaeli E, Fan S (2008) Tungsten black absorber for solar light with wide angular operation range. Appl Phys Lett 92(21):211107. https://doi.org/10.1063/1.2936997

27. Roos A, Georgson M, Wäckelgård E (1991) Tin-oxide-coated anodized aluminium selective absorber surfaces I. Preparation and characterization. Sol Energy Mater 22(1):15-28. https://doi. org/10.1016/0165-1633(91)90003-4

28. Rushing $\mathrm{H}$, Karl A, Wisnowski J (2014) Design and analysis of experiments by Douglas montgomery: a supplement for using JMP. SAS Institute, Cary

29. Shimizu M, Kohiyama A, Yugami H (2015) High-efficiency solarthermophotovoltaic system equipped with a monolithic planar selective absorber/emitter. J Photonics Energy 5(1):053099. https://doi.org/10.1117/1.JPE.5.053099
30. Standard ASTM (2007) Standard tables for reference solar spectral irradiances: direct normal and hemispherical on $37^{\circ}$ tilted surface. Society for Testing Matls, West Conshohocken, PA

31. Tian Y, Ghanekar A, Liu X, Sheng J, Zheng Y (2018) Tunable wavelength selectivity of photonic metamaterials-based thermal devices. J Photon Energy 9(3):032708. https://doi.org/10.1117/1. JPE.9.032708

32. Tian Y, Zhao CY (2013) A review of solar collectors and thermal energy storage in solar thermal applications. Appl Energy 104:538-553. https://doi.org/10.1016/j.apenergy.2012.11.051

33. Valizade $M$, Heyhat $M$, Maerefat M (2020) Experimental study of the thermal behavior of direct absorption parabolic trough collector by applying copper metal foam as volumetric solar absorption. Ren Energy 145: 261-269. https://doi.org/10.1016/j. renene.2019.05.112

34. Wang H, Chang JY, Yang Y, Wang L (2016) Performance analysis of solar thermophotovoltaic conversion enhanced by selective metamaterial absorbers and emitters. Int J Heat Mass Transfer 98:788-798. https://doi.org/10.1016/j.ijheatmasstrans fer.2016.03.074

35. Wu C, Neuner B III, John J, Milder A, Zollars B, Savoy S, Shvets $\mathrm{G}$ (2012) Metamaterial-based integrated plasmonic absorber/ emitter for solar thermo-photovoltaic systems. J Opt 14(2):024005. https://doi.org/10.1088/2040-8978/14/2/024005

Publisher's Note Springer Nature remains neutral with regard to jurisdictional claims in published maps and institutional affiliations. 